\title{
Habitual Size and Projective Size: The Logic of Spatial Systems in Children's Drawings
}

\author{
C. Lange-Küttner \\ London Metropolitan University and University of Bremen
}

\begin{abstract}
The current study analyzed figure size modification in different types of spatial context (C. LangeKüttner, 1997, 2004) for sequence and practice effects. Children of 7, 9, and 11 years of age, as well as 17-year-olds, drew figures in a series of ready-made spatial axes systems, which (a) logically increased in dimensional complexity as in child development, (b) were randomized in sequence, or (c) were absent, as a control condition for figure size reduction through practice. Already 7-year-olds could subtly adapt figure size in the logical sequence, but the amount of size reduction stayed within the same size range as in the other two conditions. Only at 9 years of age did children show sensitivity to spatial constraints, with smaller figures in both the logical and random sequence than in the control condition. At 11 years of age, the spatial scale of figure size was maximized, particularly so in children who could change perspective and construct a bird's-eye view, whereas this effect was attenuated in the 17-year-olds. Implications of the results for domain-specific conceptual development are discussed.
\end{abstract}

Keywords: pictorial space, size modification in axes systems, spatial constraints and possibilities, conceptual development, developmental transitions

Children's drawings as data about internal representations have a long and rich tradition and have largely followed the methodological and theoretical trends in developmental psychology. There are longitudinal studies of individual children, the measurement of the amount of detail in the human figure drawing served as an index of intelligence that was found to be highly correlated with psychometric tests until adolescence, and more recently domain-specific analyses of children's planning and modification strategies of graphic shapes until they reflect views (see review by Lange-Küttner \& Vinter, 2008). The notion of the graphic schema dates back to Luquet (1927; Luquet, 1927/2001) and was widely drawn upon by Piaget and Inhelder (1956) in their work on the development of the space concept. There is a wide consensus in the literature that the emergence of visually realistic images in chil-

C. Lange-Küttner, School of Psychology, Faculty of Life Sciences, London Metropolitan University, London, United Kingdom, and Faculty of Human and Health Sciences, University of Bremen, Bremen, Germany.

Parts of this research were presented in the workshop "Productivity in Pictorial Space" at the 12th European Conference for Developmental Psychology. The collection of drawings in England were organized by Enno Küttner at Claregate Primary School, Woodthorne Primary School, and Wolverhampton Grammar School, West Midlands, England. Louis Stott and Enno Küttner measured figure sizes; controls were carried out by the author. The drawings in Scotland were collected at Scotstown School, Aberdeen, Scotland, by Alyson Flett and Patricia Chalmers, who also carried out the figure size measurements. Barbara Henderson and the author carried out control measurements of all Scottish drawings. The Scottish part of the study was conducted while the author was at the University of Aberdeen, Aberdeen, Scotland.

Correspondence concerning this article should be addressed to C. Lange-Küttner, London Metropolitan University, Faculty of Life Sciences, School of Psychology, 1, Old Castle Street, London E1 7NT, United Kingdom. E-mail: c.langekuettner@londonmet.ac.uk dren's drawings is a problem-solving process of adapting the production of a graphic schema to visual perception (e.g., Freeman, 1980; Kosslyn, Heldmeyer, \& Locklear, 1980; Willats, 2005). However, the idea that children would adapt their schemata to those of their more advanced peers in a more socially guided process has also been proposed (Wilson \& Wilson, 1982). In any case, children's modification of the early graphic schemata, until they become informative about specific views, is a rather slow process in middle childhood, a reason why Van Sommers (1984) called the persistence of existing drawing habits children's "conservatism." This inflexibility, or rigidity, has been an important research topic in recent years in the drawing literature (e.g., Barlow, Jolley, White, \& Galbraith, 2003; Karmiloff-Smith, 1990, 1995; Picard \& Vinter, 1999, 2007; Vinter, Picard, \& Fernandes, 2008; Zhang, Thomas, \& Robinson, 1997). However, the issue of flexibility has always been plagued by the question of whether children actually prefer one way of drawing over another, that is, whether this conservatism is a choice or an inability. Some authors have found that young children may actually prefer intellectual over visual realism because of the unambiguous view on objects (Davis, 1983, 1985; Light \& Nix, 1983; Mitchell \& Taylor, 1999; Moore, 1986). Vinter and Marot (2007) pointed out that young children can indeed produce many visually realistic features on demand.

One main endeavor when trying to achieve a visually realistic drawing is to improve visual appearance with respect to dimensionality, that is, the creation of a three-dimensional impression on the two-dimensional surface of the drawing sheet, in other words, in pictorial space. This can be done quite efficiently by structuring the pictorial space with spatial axes systems, also called drawing systems (Dubery \& Willats, 1972; Lange-Küttner, 1989, 1994; Willats, 1985, 1997). The emergence of explicit pictorial space systems affects the depiction of contour and size (Deregowski, 
2008; Deregowski \& Dziurawiec, 1996; Lange-Küttner, 2008c; Lange-Küttner, Kerzmann, \& Heckhausen, 2002; Lange-Küttner \& Reith, 1995; Nicholls, 1995; Reith, 1988). When children draw specific object features, the amount of detail they can depict is confounded with size (Silk \& Thomas, 1986). A good example of this is the portrait, which captures individual detail of a person but often enlarges the figure so much that only half a figure, or just the head, can fill the canvas, whereas in a perspective landscape painting, people are necessarily drawn smaller, as many must fit onto one page. Kosslyn (1994, p. 368) compared this object size modification to a zoom in a camera. Size can indeed be a more powerful predictor of depth than the angularity of the spatial axes themselves (Deregowski \& Parker, 1996) or than distance (Ebersbach \& Resing, 2007).

In children's drawings, previous research demonstrated that the higher the number of figures drawn by children, the smaller they had to be. However, the spatial axes systems also affected figure size, that is, the more elaborate the space system, the smaller the figures were. These two factors impacting on figure size were quite unrelated and, hence, one was called object-driven size modification, and the second was called axes-driven size modification (Lange-Küttner, 2004).

Object-driven size regulation between two figures is mastered at about 5 years of age, when objects become modified in relation to each other in a realistic fashion, that is, children depict more realistic size differences for adjacent figures such as a dog and the owner (Silk \& Thomas, 1986, 1988). The unrealistic figure sizes drawn by young children appear to result from an apparently universal preference in children to start large in drawing (Freeman, 1980; Thomas, 1995). Space is allocated on a first-come, firstserved basis by younger children, that is, once the first shape is drawn large, less pictorial space is left over for the additional ones. Hence, one can conclude that the more figures or objects that are drawn in a space and the later they are drawn, the smaller they will be. Object-driven size modification in spatial axes systems of varying complexity was still a powerful factor in 7- and 9-yearolds, but axes-driven size modification proved to be the only significant predictor at 11 years of age (Lange-Küttner, 1997, 2004, 2008b). It is not clear, however, how this change toward a more powerful axes space system could have occurred. In the previous research, children were either constructing the spatial system themselves, or the ready-made spatial axes systems were sorted in a "logical" sequence, which simulated the developmental acquisition sequence. But would not a true understanding of a spatial system be demonstrated only if children produced the same size modifications in the ready-made spatial axes systems when in random sequence, where their attention would not be drawn into the gradual unfolding of dimensionality?

Initially, children draw figures only in empty space, and hence they draw three-dimensionally usually earlier when drawing these objects than when structuring the whole page (Lange-Küttner, 1994, 2008c). In single objects, such as cubes, the third dimension gradually unfolds in relatively distinctive steps (e.g. Bremner, Morse, Hughes, \& Andreasen, 2000; Caron-Pargue, 1992; Cox, 1986; Mitchelmore, 1978; Willats, 1985, 1997). Initially, a cube is drawn as just one square form, an abbreviated shape without any explicit indication of three-dimensional volume (as illustrated in Figure 1, Stage 1). In a first step of unfolding the third dimension, sides of the cube are added (Figure 1, Stage 2). Sometimes all six

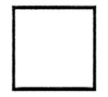

1

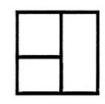

2

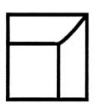

$3 \mathrm{~A}$

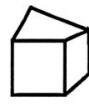

3B

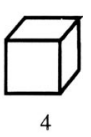

Figure 1. Unfolding of the third dimension in the cube drawing. Adapted from "Developmental Stages in Children's Representation of Regular Solid Figures" by M. C. Mitchelmore, 1978, Journal of Genetic Psychology, 133(2), p. 235. Reprinted with permission of the Helen Dwight Reid Educational Foundation. Published by Heldref Publications. Copyright (c) 1978.

sides are specified in what has been referred to as a diagrammatic layout (Kosslyn et al., 1980), and sometimes only a second or a third side is added in an object-specific way (Figure 1, Stage 3A). Thereafter, these added sides of the cube become depicted with diagonal lines, which converge toward a viewpoint outside the cube itself, in a view-specific way (Figure 1, Stage 3B). During this phase, shapes are frequently compromises between orthogonal and diagonal graphic constructions until the correct threedimensional form is achieved (Figure 1, Stage 4).

The explicit unfolding of the third dimension in the entirety of pictorial space has a slightly different sequence, as the starting point is not a self-drawn shape but a spatial axis. Such drawings might need more conceptual effort because of the higher working memory load required for processing spatial relations (Case, Stephenson, Bleiker, \& Okamoto, 1996; Dennis, 1992; Morra, 2008) or because instead of the primary unit-based strategy, frame-based strategies must be used (Vinter \& Marot, 2007). In a first step to denote spatial dimensions, a ground line is drawn (horizontal axis system). Thereafter, entire spatial fields become constructed from a combination of vertical and horizontal axes (orthogonal axes system), which finally are depicted in viewpoint perspective with the use of diagonals.

Few children or adults construct viewpoint perspective (Hagen, 1985; Lange-Küttner, 1994, 1997). Especially when younger (Hargreaves, Jones, \& Martin, 1981) or inexperienced in drawing (Deregowski, 1978, 1980), explicit spatial axes systems would not be constructed at all. In a longitudinal study (Lange-Küttner, 1994), 7- to 12-year-old rural children never drew viewpoint perspective, and only about $10 \%$ of urban children constructed viewpoint perspective at 12 years of age. A substantial proportion, one third of the rural and half of the urban children in that study, drew orthogonal axes systems, that is, spatial axes were crossed at an angle of 90 degrees, but not at oblique angles as in viewpoint perspective. Several studies (Kosslyn et al., 1980; Olson, 1970/ 1996; Perner, Kohlmann, \& Wimmer, 1984; Piaget \& Inhelder, 1956; Piaget, Inhelder, \& Szeminska, 1960) showed that acquisition of diagonality poses a much more difficult problem than does acquiring orthogonality.

The sensorimotor component appears to be no hindrance to constructing projective spatial systems, as the drawing of the straight lines of angular forms is much easier than drawing perfectly round geometric circles or the irregular, natural contour of living things (Lange-Küttner, 1998; Lange-Küttner et al., 2002). In orthogonal fields, angles always meet at $90^{\circ}$ degrees and, hence, meeting points of lines are simpler to predict than the many possible axes combinations of acute and obtuse angles necessary for viewpoint perspective. Using a drawing series with ready- 
made, predrawn axes systems (Lange-Küttner, 2004; see Figure 2) as external visual cues, which simulated this developmental acquisition sequence, made it possible to investigate the amount of figure size reduction in viewpoint perspective, independently of children's proclivity to draw projective spatial systems themselves.

In previous research on object size modification in axes systems, children drew larger and more varied figure sizes in empty space and in ground line constructions but a much smaller and more uniform figure size in orthogonal and diagonal axes systems, indicating understanding of projective pictorial space (LangeKüttner, 2004). This was the case in 7-year-olds and was only more pronounced in 11-year-olds.

Did this imply that by 7 years of age children would understand all spatial systems, including projective pictorial space long before they could actually show this in their drawings or verbal reports? In the current study, this hypothesis was tested in a more stringent experimental design with three conditions. Children were given the ready-made spatial axes systems in a logical sequence as in the previous study, where complexity of space systems gradually increased, simulating the developmental acquisition sequence. In a second condition, these space systems were presented in a randomized sequence. It was predicted that if children have a robust and powerful spatial concept, it would withstand the randomization and there would be no difference between the logical and the random sequence. However, if children's spatial concept is not robust, the random sequence would confuse and produce random size and higher figure size variability.

In a third condition, figure drawings were repeated on empty pages without ready-made axes systems to control for practice effects. This condition was included because practice alone could provide sensorimotor training, which might allow children to gradually draw smaller figures at all ages (Vinter \& Perruchet, 2000, 2002). Because drawing sheets in the practice condition had fewer spatial constraints for drawing figures than did those in either the logical or the random sequence condition, it was expected that

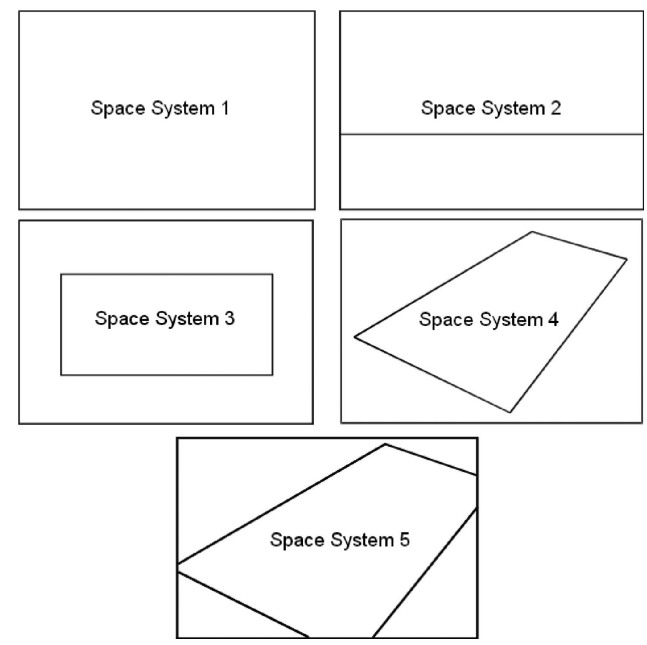

Figure 2. Unfolding of the third dimension in the ready-made drawing axes systems of the drawing series. Space System 1: Empty space; Space System 2: Horizontal axes system; Space System 3: Orthogonal axes system; Space System 4: Closed diagonal axes system; Space System 5: Open diagonal axes system. children would draw larger figures, show more size variability, and exhibit less size reduction during the drawing series.

\section{Method}

\section{Participants}

Children were from three schools in Wolverhampton, West Midlands, England, and from one school in Aberdeen, Scotland, in lower and upper middle class areas. The Scottish children were all Caucasian, whereas in the English schools, some children were from a mainly Asian British-born background. Mean ages of the sample are specified in Table 1. Age groups were labeled 7-yearolds, 9-year-olds, 11-year-olds, and 17-year-olds.

Each drawing booklet involved drawing 25 human figures at play ( 5 per drawing sheet). Not all of the mostly primary schoolage children managed to complete the series. In the practice condition, 23 children did not complete the drawing series: three 7-year-olds, twelve 9-year-olds, and eight 11-year-olds (but no 17-year-olds). In the random condition, 14 children did not complete the drawing series: one 7-year-old, ten 9-year-olds, and four 11-year-olds (but no 17-year-olds). In the logical condition, only four children did not complete the drawing series: two 7-year-olds and two 9-year-olds (but no 11-year-olds or 17-year-olds). The noncompletion rates indicated that the random sequence and especially the practice condition were more demanding than the logically presorted sequence of space systems for the children than for the late adolescents, who were not as readily "running out of steam" (Lange-Küttner, 2008a) when drawing 25 figures. These incomplete booklets were excluded from the analyses. One child was absent after a break, and seven drawing booklets contained no date of birth; thus, these drawing booklets were excluded. Furthermore, 18 drawings sets had sheets with fewer than three figures (pairs and single figures) in one or more drawings of the series and were thus excluded from analyses. This resulted in a final sample size of $N=297$ children, which was deemed sufficient for the purpose of this study. A total of 122 boys and 175 girls participated; in the logical sequence condition, there were 45 girls and 53 boys; in the random sequence condition, there were 37 girls and 62 boys; and in the practice condition, there were 40 girls and 60 boys.

\section{Apparatus}

Material. For both samples, the ready-made axes systems were printed on large paper formatted $41.5 \times 29.5 \mathrm{~cm}$ (landscape format, size A3). The drawing sheets were collated in a booklet. There were three types of booklets: (a) one with axes systems in the same order as in the typical developmental acquisition sequence, (b) one arranged in a random sequence produced according to Latin square (Fisher \& Yates, 1963, p. 86), and (c) one without ready-made axes systems, that is, just plain white paper was used as a control condition to investigate the effects of mere practice.

An illustration of the drawing systems is displayed in Figure 2. Space systems are labeled by number: $1=$ empty page (implicit space); 2 = ground line (horizontal axes); $3=$ playing field (orthogonal axes); 4 = playing field in closed perspective (diagonal axes); $5=$ playing field in open perspective (extending 
Table 1

Participant Description per Condition and Age Group

\begin{tabular}{|c|c|c|c|c|c|}
\hline Condition/Age & $n$ & $M$ & Minimum & Maximum & $S D$ \\
\hline \multicolumn{6}{|l|}{ Logical sequence } \\
\hline 7 years & 31 & 7 years, 6 months & 7 years, 1 month & 8 years, 0 months & 4 months \\
\hline 9 years & 21 & 9 years, 6 months & 8 years, 11 months & 10 years, 0 months & 4 months \\
\hline 11 years & 23 & 11 years, 7 months & 10 years, 11 months & 12 years, 0 months & 4 months \\
\hline 17 years & 23 & 17 years, 4 months & 16 years, 8 months & 17 years, 8 months & 4 months \\
\hline Total & 98 & & & & \\
\hline \multicolumn{6}{|l|}{ Random sequence } \\
\hline 7 years & 28 & 7 years, 9 months & 7 years, 3 months & 8 years, 4 months & 4 months \\
\hline 9 years & 24 & 9 years, 8 months & 9 years, 1 month & 10 years, 8 months & 5 months \\
\hline 11 years & 27 & 11 years, 11 months & 11 years, 2 months & 13 years, 1 month & 4 months \\
\hline 17 years & 20 & 17 years, 6 months & 17 years, 2 months & 17 years, 9 months & 2 months \\
\hline Total & 99 & & & & \\
\hline \multicolumn{6}{|l|}{ Practice control } \\
\hline 7 years & 35 & 7 years, 9 months & 7 years, 2 months & 8 years, 4 months & 4 months \\
\hline 9 years & 26 & 9 years, 11 months & 9 years, 3 months & 10 years, 8 months & 5 months \\
\hline 11 years & 20 & 11 years, 8 months & 11 years, 2 months & 12 years, 8 months & 5 months \\
\hline 17 years & 19 & 17 years, 4 months & 17 years, 0 months & 17 years, 9 months & 3 months \\
\hline Total & 100 & & & & \\
\hline
\end{tabular}

diagonal axes). The numbers of the space systems were used in the text when reporting results from post hoc comparisons among conditions per space system and on the $x$-axis of the plotted means. Space System 1 consisted of "empty space," that is, a page with no explicit spatial axes, where implicit object alignments could occur (Light \& Humphreys, 1981; Light \& MacIntosh, 1980). Space System 2 contained a horizontal axis, also called a ground line, running $10 \mathrm{~cm}$ from the lower edge. Space System 3 consisted of an orthogonal playing field, which could, for instance, be used to represent a football pitch or a swimming pool. Edges of the rectangular field were located $7 \mathrm{~cm}$ from the upper and lower rim and $5 \mathrm{~cm}$ from the left and right rim. Space System 4 consisted of a playing field with about the same area as the third sheet but twisted into a diagonal perspective. Finally, Space System 5 consisted of an open diagonal playing field, with a larger playing field because spatial axes were projecting beyond the actual drawing sheet, converging outside the paper at an angle of $40^{\circ}$ degrees. Hence, whereas the closed diagonal playing field constituted another view on the same field as the orthogonal playing field, the open diagonal playing field represented perspective in a stronger sense, where vectors could point into a potentially unlimited extension of space.

English children $(n=219)$ used pencils and colored pencils, whereas Scottish children $(n=78)$ used crayons, which were part of their school equipment. It could have been possible that crayons would produce a larger figure size. Although this was not expected to have an impact on the within-participant effects of the drawing series, as the same material was used throughout, it could have contributed to a between-participant effect of size, particularly because Scottish children's drawings were not equally distributed across conditions. For this reason, although this factor was irrelevant to the experimental design of the study as such, this regional difference in drawing material was partialed out as a covariate in the analyses of variance, and the means plotted in the figures are the adjusted values.

Instructions. Instructions were the same across age groups. Instructions were printed on an extra page in the booklets before the respective drawing sheet. Instructions were as follows in the logical and random sequence conditions: for the empty sheet, "Please draw yourself and four friends playing a ball game"; for the ground line, "We have drawn a line for you on the page. Please start from there and draw yourself and four friends playing a ball game"; for the orthogonal area, "Now we have drawn a playing field for you. Please start from there and draw yourself and four friends playing a ball game"; for the closed diagonal area, "This time we have drawn a playing field for you as it looks when you are standing in front of it. Please start from there and draw yourself and four friends playing a ball game"; and for the open diagonal area, "This time we have drawn a playing field for you as it looks when you are standing in front of it. Please start from there and draw yourself and four friends playing a ball game."

In the practice control condition (all empty pages without predrawn axes systems), the first instruction was the following: "Please draw yourself and four friends playing a ball game." The instruction for the subsequent four pictures was as follows: "Once more, please draw yourself and four friends playing a ball game." The instruction for the final fifth picture was given as "One last time, please draw yourself and four friends playing a ball game."

\section{Procedure}

Booklets were placed in front of the children. Date of birth and gender were noted on the front cover of the drawing booklet by the teacher or the university technician. Children read the instructions by themselves, as in the United Kingdom, children are schooled between 4 and 5 years of age and are usually fluent readers by the age of 7 years. Otherwise, drawings were collected anonymously, without names. Participants produced the drawing in the classroom with sufficient space between tables to avoid copying.

\section{Scoring}

The size of all figure drawings was measured in centimeters. Human figure drawings were not included when only a partial 
figure was drawn, unless it was a full-sized half figure, but this was rare. In these cases, the remaining three or four figures were averaged to a picture size score.

Figures were almost always drawn singly, and no overlap occurred that could have made size measurement difficult. The size of the human figure drawings was measured in centimeters with a ruler. The scoring instruction was to measure the human figure with a ruler from the parting of the hair to the foot sole. This was carried out for each figure by drawing a horizontal axis over the head and an axis (platform) underneath the feet and then measuring the vertical distance in between these two horizontal axes. When legs were of unequal length, resulting in a tilted platform, size was measured along the body axis through the midpoint from where the legs emerged, down to the platform, which was approximately the middle point between the two feet. This was also applicable when the figure was depicted in side view, as the body axis would be measured along the back. In cases when figures were drawn as from above, in bird's-eye view, the same rules applied. Upright standing hair was included as belonging to the head, but hats were not. Studs and heels were excluded as being distinct from the sole of the shoe. Given the trace of a pencil, a divergence in measurement of more than $2 \mathrm{~mm}$ was taken as measurement error; for a crayon, a divergence in measurement of more than $4 \mathrm{~mm}$ was taken as measurement error. All Scottish drawings were measured by two university technicians, and all drawings were double-checked by a third technician and the author, with corrections rarely necessary.

Size measurement of the English sample of drawings was shared between a university technician and a psychologist. To establish reliability, a representative number of sets was drawn from each age group and condition by the author. Each booklet contained $5 \times$ 5 figures $=25$ figures, but some had 4 figures over which size was averaged. Hence, percentages of the representative booklets are fairly precise estimates. A similar number of figures was controlled per condition: In the logical sequence condition, reliability was $98.6 \%$ based on double measurement of 713 figures (approximately $28.5 \%$ ). In the random sequence condition, $98.4 \%$ reliability was obtained based on measurement of 649 figures (approximately $41.0 \%$ ). In the practice condition, reliability was $94.7 \%$ based on 767 figures (approximately 50\%). This latter reliability between $90 \%$ and $95 \%$ would have been quite acceptable, but as it was not as excellent as in the other two conditions, all drawings in the practice condition were double measured and scores were corrected.

\section{Results}

Sizes of human figures in centimeters were averaged per participant per picture with SPSS and analyzed with analyses of variance (ANOVAs). Drawing scores of the randomized sequence were sorted into the Space System 1-5 sequence for analyses and data plotting. Vertical bars in the figures represent standard error. When Mauchly's test of sphericity was significant, degrees of freedom were adapted with the Huynh-Feldt method. The Scheffé test $(p<.05)$ was used as a post hoc test for between-participants group differences among the three experimental conditions.

\section{Average Figure Size}

Because 27 children were observed to draw a top-down bird'seye view on heads of players in Space System 3, with the orthogonal playing field (see Figures 2 and 5), this was tested statistically. Chi-square analysis showed that the incidence of drawing a bird's-eye view in the orthogonal playing field increased with age, $\chi^{2}(3, N=297)=35.26, p<.001$, phi $=.35: 1.1 \%$ of the sample $(n=1)$ for 7 -year-olds, $0 \%$ of the sample $(n=0)$ for 9 -year-olds, $15.7 \%$ of the sample $(n=11)$ for 11 -year-olds, and $24.2 \%$ of the sample $(n=15)$ for 17-year-olds. Hence, the drawing of bird'seye views was controlled in the following analyses, especially in the two older age groups.

A 4 (age group) $\times 5$ (drawing series) $\times 3$ (condition) ANOVA with repeated measures was carried out, with drawing series as a within-subject factor and region as a covariate. All quoted and plotted means are corrected for the covariate. The covariate was significant, $F(1,297)=19.95, p<.001, \eta^{2}=.07$, with children using crayons $(M=7.04 \mathrm{~cm})$ drawing larger than children using pencils $(M=5.29 \mathrm{~cm})$. As expected, there was no significant interaction of the covariate with size modification in the different spatial axes systems, $F(4,297)=1.58, p=.18$, as children used either the crayons or the pencils throughout the drawing series.

There was a main effect of age, $F(3,297)=3.05, p<.05, \eta^{2}=$ .03 , as participants drew, on average, increasingly smaller figures with age: that is, in 7-year-olds, $M=6.05 \mathrm{~cm}$; 9-year-olds, $M=5.92$ cm; 11-year-olds, $M=5.81 \mathrm{~cm}$; and 17-year-olds, $M=5.05 \mathrm{~cm}$.

The within-subject factor of drawing series was significant as a main effect, $F(3.58,297)=2.66, p<.05, \eta^{2}=.01$, and there was an interaction between age and drawing series, $F(8,297)=4.44$, $p<.001, \eta^{2}=.05$. Importantly, the experimental condition (logical vs. random vs. practice) was significant as a main effect, $F(2,297)=11.50, p<.001, \eta^{2}=.08$, in a two-way interaction with drawing series, $F(8,297)=8.53, p<.001, \eta^{2}=.06$, and all of these effects combined in a three-way interaction of condition, age, and drawing series, $F(24,297)=3.41, p<.001, \eta^{2}=.07$. Running the model without the 27 bird's-eye view drawers slightly reduced the significance of this interaction, $F(24,270)=1.96, p<$ $.01, \eta^{2}=.04$, but did not abolish it. To decompose the robust three-way interaction, the ANOVA was repeated for each age group.

7-year-olds. In the youngest age group, the effect of the covariate was significant, $F(1,94)=4.58, p<.05$, with 7-year-old children drawing larger with crayons $(M=6.91 \mathrm{~cm})$ than with pencils $(M=5.77 \mathrm{~cm})$. As predicted, the interaction of region with the drawing series was not significant, $F(1$, 94) $=1.07, p=.37$.

The main effects of drawing series and condition were not significant $(p s<.64)$. The interaction of drawing series with condition was marginally significant, $F(7.31,94)=1.99, p=$ $.052, \eta^{2}=.04$, as 7 -year-olds constantly reduced figure size only in the logical sequence (see Figure $3 \mathrm{~A}$ ). No single post hoc test was significant. Thus, whereas figure size reduction occurred only in the logical sequence, this took place only within the centimeter range of the other two conditions at 7 years.

9-year-olds. In the 9-year-old group, the covariate region was significant, $F(1,71)=7.40, p<.01, \eta^{2}=.10$, with children drawing larger figures with crayons $(M=7.46 \mathrm{~cm})$ than with pencils $(M=5.34 \mathrm{~cm})$. As predicted, this effect was 
A

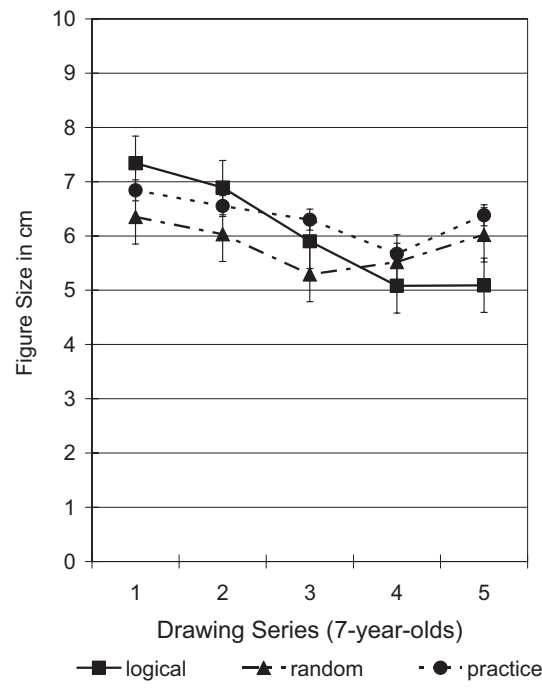

C

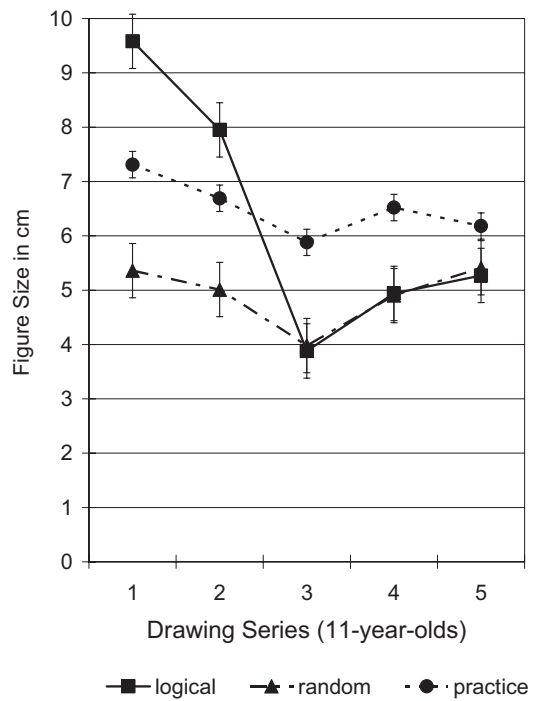

B

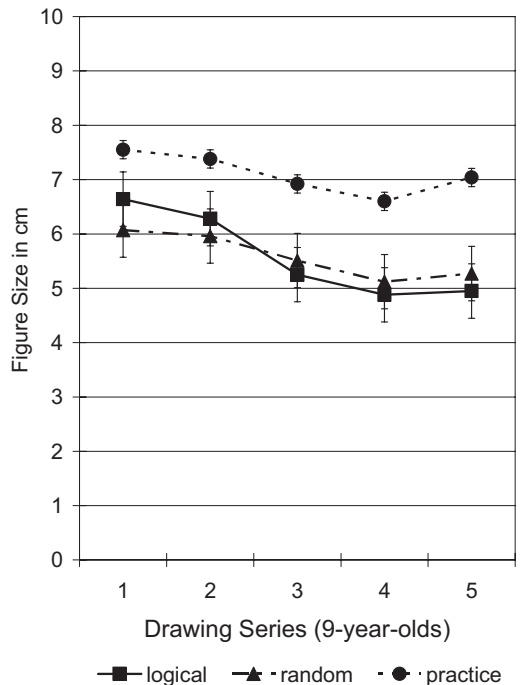

D

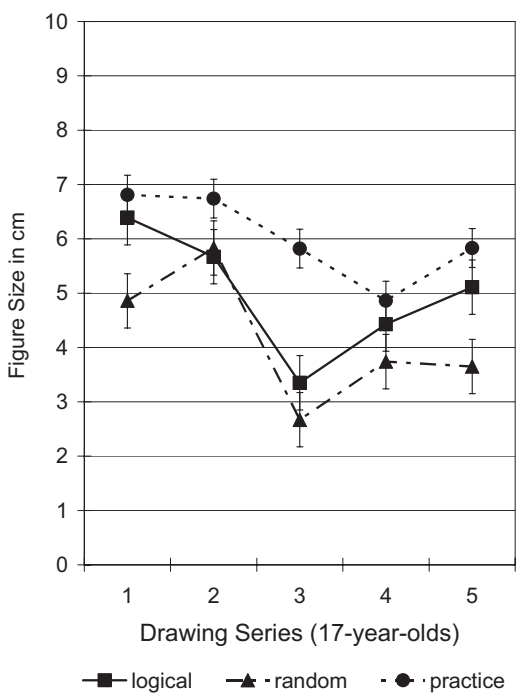

Figure 3. Figure size modification. Plotted means of the three-way interaction between age groups, drawing series, and condition. (A) 7-year-olds reduced figure size only in the logical condition but within the average size of the other two conditions. (B) 9-year-olds drew larger figure size in the practice control condition than in the axes systems, indicating an emerging sensitivity to spatial constraints. (C) 11-year-olds used a large spatial scale to expand figures in empty space and reduce figure size in more constrained space systems. (D) 17-year-olds showed attenuated effects of size expansion in systems with few spatial constraints.

not significant in interaction with the drawing series, $F(4,71)=$ $0.38, p=.82$.

Condition was significant, $F(2,71)=3.67, p<.05, \eta^{2}=.10$ (see Figure 3B). Post hoc tests showed that average size in the practice condition was now larger than in the logical sequence (Space Systems 2, 3, 4, and 5), except on the first page (Space System 1). Average size was larger in the practice than in the random condition only in the perspective systems (Space Systems 4 and 5). The interaction between drawing series and condition was not significant, $F(2,71)=0.74, p=.65$. Thus, at 9 years of age, a new, though rather general, sensitivity to spatial constraints emerged: The practice condition, that is, drawing in empty space without any spatial constraints, was now likely to elicit, on average, larger figure drawings than the ready-made axes systems. This was not the case at 7 years.

11-year-olds. Among 11-year-olds, the covariate was significant, $F(1,70)=8.24, p<.01, \eta^{2}=.11$, with children using crayons $(M=6.73 \mathrm{~cm})$ drawing larger than children using pencils 
$(M=5.38 \mathrm{~cm})$. As predicted, the covariate was not significant in interaction with the drawing series, $F(4,70)=1.24, p=.30$.

The main effects of the drawing series, $F(3.09,70)=2.69, p<$ $.05, \eta^{2}=.04$, and condition, $F(2,70)=5.62, p<.01, \eta^{2}=.15$, were significant and interacted with each other, $F(8,70)=10.63$, $p<.001, \eta^{2}=.24$ (see Figure 3C). Post hoc tests showed that in the logical sequence, average size was larger in empty space and the horizontal axes system (Space Systems 1 and 2, respectively), but smaller in all crossed systems (Space Systems 3, 4, and 5), compared with the practice condition. Thus, in 11-year-olds, size reduction in the logical sequence differed either upward or downward from the practice condition in all spatial systems. In the random sequence, average size was continuously smaller than in the practice condition in all spatial systems except the last one (i.e., size decrements were observed in Spatial Systems 1, 2, 3, and 4). Furthermore, 11-year-olds now produced nearly identical figure size in the orthogonal and diagonal axes systems in both the random and logical sequence conditions.

When the model was run without the bird's-eye view drawers, the main effect of size reduction in the drawing series became stronger, $F(3.14,59)=27.59, p<.001, \eta^{2}=.33$, whereas the effects of the experimental condition, $F(2,59)=3.58, p<.05$, $\eta^{2}=.11$, and the interaction with size reduction were a little less pronounced, $F(8,59)=6.53, p<.001, \eta^{2}=.19$. Figure 4A shows that average figure size on the first, empty sheet in the logical sequence and the practice condition were now much more similar, which one would have expected given that both groups had one empty page at the beginning of the drawing series. The bird's-eye view drawers were apparently a group with great skill in size modification, depicting a top-down view with diminished size as well as enlarging figure size in empty space.

What appeared to be different from the 7- and 9-year-olds, however, was that 11-year-olds seemed to subtly increase size in the crossed axes systems, that is, the orthogonal, the closed diagonal, and the open diagonal perspective systems. In order to test this size increase only, two one-factorial repeated measurement ANOVAs were conducted for (a) the orthogonal compared with the closed diagonal perspective system and (b) the closed compared with the open diagonal perspective, respectively, excluding the subsample of the practice condition. ANOVA models of variance had the two respective space systems as within-subject factor, bird's-eye view as a between-subjects variable, and region as a covariate. In the first ANOVA, children who used crayons drew larger than those drawing with pencils, $F(1,50)=7.21, p<.01$, $\eta^{2}=.13$, but this did not interact with the task effect $(p>.50)$. More importantly, and as expected, bird's-eye view drawers drew figures of a smaller average size (bird's-eye view $M=3.11 \mathrm{~cm}$; no bird's-eye view $M=4.62 \mathrm{~cm}), F(1,50)=6.41, p<.05, \eta^{2}=.12$. The 11-year-olds drew smaller average figure size in the orthogonal than in the perspective system, $F(1,50)=10.10, p<.01$, $\eta^{2}=.18$, but this was particularly pronounced in the bird's-eye view drawers, $F(1,50)=18.46, p<.001, \eta^{2}=.28$ (for bird's-eye view drawers, Spatial System $3 M=1.59 \mathrm{~cm}$ and Spatial System $4 M=4.63 \mathrm{~cm}$; while for no bird's-eye view drawers, Spatial System $3 M=4.35 \mathrm{~cm}$ and Spatial System $4 M=4.88 \mathrm{~cm}$ ). As bird's-eye view drawers drew very small sizes in the orthogonal spatial field, it is not surprising that they were more likely to draw larger when drawing the full figure view again in the perspective systems. In the second ANOVA, no significant task effects were found $(p s>.34)$. This implies that the apparent increase of figure size in the perspective systems was not significant. As an illustration of the comparison of bird's-eye view drawers versus non-bird's-eye view drawers in the orthogonal versus perspective system, refer to Figure 5. The illustrations show that whereas bird's-eye view drawers drew inadvertently small in the orthogonal system, this could also be the case in the remaining sample, where children could draw either small or large.

17-year-olds. There were no participants of this age group who used crayons.
A

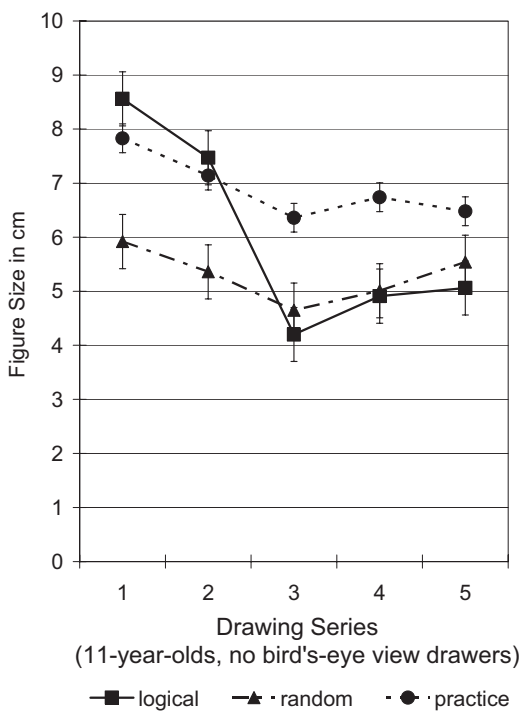

B

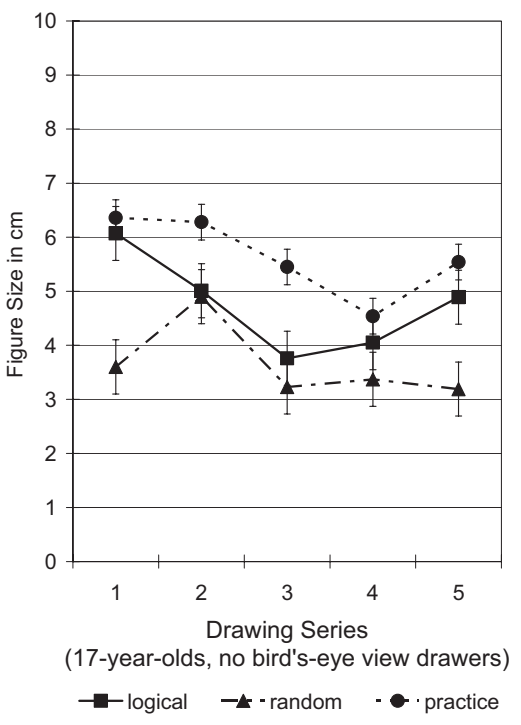

Figure 4. Figure size modification without the bird's-eye view drawers. (A) 11-year-olds and (B) 17-year-olds. 


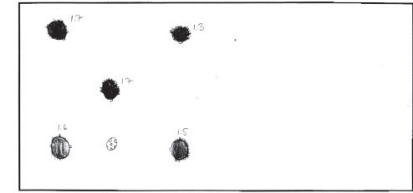

Bird's eye view in the orthogonal system

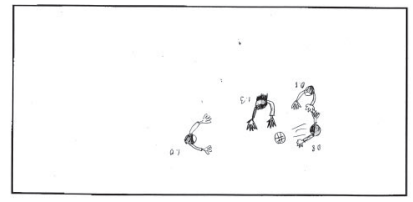

Bird's eye view in the orthogonal system

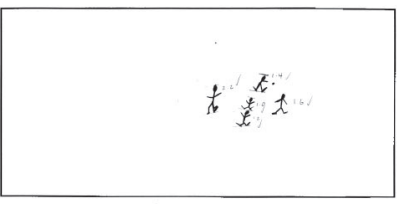

Full view in the orthogonal system

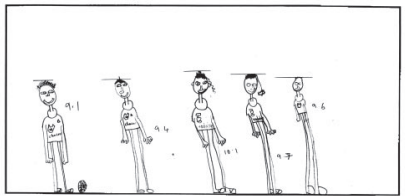

Full view in the orthogonal system

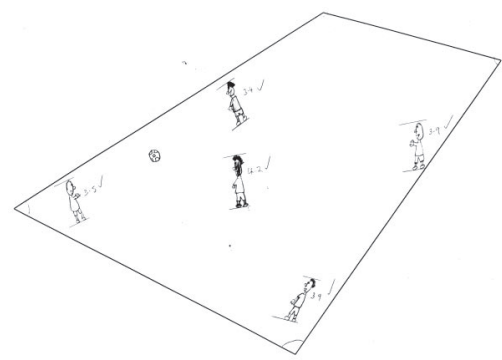

Same child, closed diagonal system

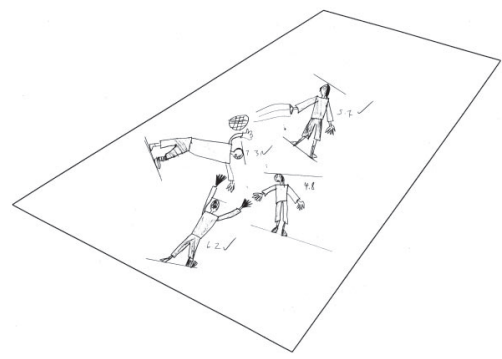

Same child, closed diagonal system

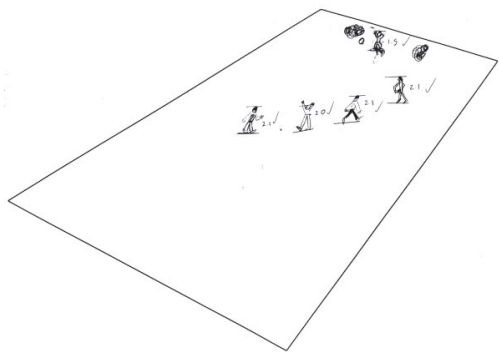

Same child, closed diagonal system

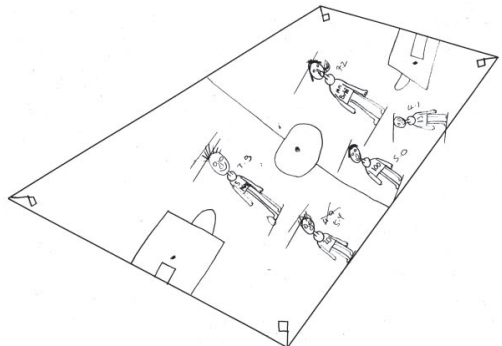

Same child, closed diagonal system

Figure 5. Eleven-year-olds: Figure size reduction in orthogonal (left side) and diagonal (right side) space systems.

The main effects of drawing series, $F(3.46,62)=23.22, p<$ $.001, \eta^{2}=.28$, and condition, $F(2,62)=4.90, p<.05, \eta^{2}=$ .14 , were significant and interacted with each other, $F(8,62)=$ $3.09, p<.01, \eta^{2}=.10$ (see Figure 4D). Post hoc tests showed that average size in the practice condition was significantly larger than in the random sequence (Space Systems 3 and 5) and the logical sequence (Space System 3). Hence, although overall the statistical effects were the same as for the 11-year-olds, differences between the experimental conditions were attenuated in the 17-year-olds.

When the bird's-eye view drawers were excluded, statistical effects were slightly reduced but still statistically significant. Ef- fect sizes stayed about the same in the size reduction effect in the drawing series, $F(3.79,47)=10.59, p<.001, \eta^{2}=.27$; the effect of experimental condition, $F(2,47)=3.40, p<.05, \eta^{2}=.13$; and the interaction of these two factors, $F(8,62)=2.21, p<.05, \eta^{2}=$ .09 (see Figure 4B).

The same tests for subtle increase in the crossed axes systems as for the 11-year-olds were carried out for the 17-year-olds. The first analysis testing the orthogonal and the closed diagonal axes system showed the same result as for the 11-year-olds, that is, 17-year-old bird's-eye view drawers drew a smaller average figure size (bird's-eye view $M=2.44 \mathrm{~cm}$; no bird's-eye view $M=3.65 \mathrm{~cm}), F(1,43)=8.19, p<.01, \eta^{2}=.17$. Moreover, 
the late adolescents drew comparably smaller average figure size in the orthogonal system, $F(1,43)=58.59, p<.001, \eta^{2}=$ .59 , and again this was particularly pronounced in the bird'seye view drawers, $F(1,43)=42.58, p<.001, \eta^{2}=.51$ (for bird's-eye view drawers, Spatial System $3 M=1.02 \mathrm{~cm}$ and Spatial System $4 M=3.87 \mathrm{~cm}$; while for no bird's-eye view drawers, Spatial System $3 M=3.54 \mathrm{~cm}$ and Spatial System 4 $M=3.76 \mathrm{~cm})$. The statistical power of the effects increased considerably in comparison with that for the 11-year-olds. When comparing closed versus open diagonal perspective, there was a trend in which the entire sample drew a larger figure size when the diagonal field was larger, $F(1,43)=42.58, p=.064$, $\eta^{2}=.08$. No other effect was significant $(p s>.69)$.

\section{Figure Size Variability}

The figure size variability score indicated how similar in size the figures were on each sheet. Size variability was predicted to be higher in the less constrained spatial systems, in particular in the practice condition, and comparably lower in the orthogonal and diagonal systems. The standard deviation between figure sizes in one drawing was computed to analyze size variability.

A 4 (age group) $\times 5$ (drawing series) $\times 3$ (condition) ANOVA with repeated measures was conducted with drawing series as a within-subject factor and crayon/pencil as a covariate. The effect of the covariate was not significant as a main effect, $F(1,297)=$ $0.22, p=.64$, but it interacted with drawing series, $F(4,297)=$ $3.20, p<.05, \eta^{2}=.01$. When children used pencils, size variability was highest in the first picture and diminished thereafter (Space System $1 M=1.34 \mathrm{~cm}$, Space System $2 M=1.04 \mathrm{~cm}$, Space System $3 M=1.00 \mathrm{~cm}$, Space System $4 M=1.07 \mathrm{~cm}$, and Space System $5 M=1.09 \mathrm{~cm}$ ). When children used crayons, size variability was highest in the horizontal axes system (Space System $1 M=1.12 \mathrm{~cm}$, Space System $2 M=1.24 \mathrm{~cm}$, Space System $3 M=1.10 \mathrm{~cm}$, Space System $4 M=1.01 \mathrm{~cm}$, and Space System $5 M=1.08 \mathrm{~cm}$ ). Thus, with both drawing materials, size variability was highest in the two space systems with the lower spatial constraints.

There was a main effect of age, $F(3,297)=3.96, p<.01, \eta^{2}=$ .04 , but age differences were unsystematic (for 7-year-olds, $M=$ $1.16 \mathrm{~cm}$; for 9-year-olds, $M=0.95 \mathrm{~cm}$; for 11-year-olds, $M=1.20$ $\mathrm{cm}$; and for 17-year-olds, $M=0.94 \mathrm{~cm}$ ), with no single post hoc test significant. Furthermore, the effect of condition, $F(2,297)=$ 9.67, $p<.001, \eta^{2}=.06$, was significant. Post hoc tests showed that variability was higher in the practice condition than in either of the two space system sequences.

Drawing series, $F(4,297)=3.88, p<.01, \eta^{2}=.01$, as well as the two-way interactions of drawing series with age, $F(12,297)=$ $1.97, p<.05, \eta^{2}=.02$, and with condition, $F(8,297)=3.65, p<$ $.001, \eta^{2}=.03$, were significant, and all of these factors interacted in a three-way interaction of condition and drawing series with age, $F(24,297)=2.04, p<.01, \eta^{2}=.04$. When the bird's-eye view drawers were excluded, this three-way interaction remained nearly identical, $F(24,270)=2.09, p<.01, \eta^{2}=.05$. Thus, as before, the ANOVA was conducted separately for each age group, with plotted means shown in Figure 6.

7-year-olds. No statistical effects reached significance for figure size variability among the 7-year-olds. 9-year-olds. Among the 9-year-olds, the covariate was not significant, $F(1,71)=0.64, p=.42$, but the interaction of region with the drawing series approached significance, $F(4,71)=2.35$, $p=.054, \eta^{2}=.03$. Size variability was initially higher in children using crayons (Space System $1 M=1.22 \mathrm{~cm}$; Space System 2 $M=1.10 \mathrm{~cm}$; Space System $3 M=1.08 \mathrm{~cm}$; Space System $4 M=$ $1.00 \mathrm{~cm}$; Space System $5 M=1.08 \mathrm{~cm}$ ) than those using pencils (Space System $1 M=0.93 \mathrm{~cm}$; Space System $2 M=0.77 \mathrm{~cm}$; Space System $3 M=0.79 \mathrm{~cm}$; Space System $4 M=0.88 \mathrm{~cm}$, and Space System $5 M=1.04 \mathrm{~cm}$ ).

A significant effect of condition, $F(2,71)=4.11, p<.05, \eta^{2}=$ .11 , showed that size variability was comparably higher in the practice condition (logical $M=0.81$, random $M=0.88$, practice $M=1.16)$. Furthermore, there was a significant effect of drawing series, $F(3.87,71)=2.83, p<.05, \eta^{2}=.04$, and a significant two-way interaction between drawing series and condition, $F(8$, $71)=2.09, p<.05, \eta^{2}=.06$ (see Figure 6B), which showed lower variability in the logical condition than in the practice condition (Space Systems 3 and 4) and also lower variability in the random sequence condition than in the practice condition (Space System 3). This demonstrated that the orthogonal and diagonal systems not only elicited smaller figure sizes, on average, but also comparably fewer differences between figures.

11-year-olds. In the 11-year-old group, the covariate was significant, $F(1,70)=7.61, p<.01, \eta^{2}=.10$, with children drawing with pencils showing more size variation $(M=1.32 \mathrm{~cm})$ than those drawing with crayons $(M=0.89 \mathrm{~cm})$. There was no interaction of the covariate with drawing series, $F(3.66,70)=0.61$, $p=.65$.

There was a main effect of condition, $F(2,70)=3.95, p<.05$, $\eta^{2}=.11$, with the least size variability in the random sequence $(M=0.97 \mathrm{~cm})$, somewhat more size variation in the logical sequence $(M=1.08)$, and the most size variation in the practice condition $(M=1.55 \mathrm{~cm})$. A significant two-way interaction of drawing series with condition, $F(7.32,70)=3.78, p<.001, \eta^{2}=$ .10 , was found. Post hoc tests showed that only in the horizontal system was size variability higher in the logical than in the random sequence. In the orthogonal system, both types of axes system sequences produced less variability than in the practice condition (see Figure 6C).

When bird's-eye view drawers were excluded from the analysis, the effect of region, $F(1,70)=8.07, p<.01, \eta^{2}=.13$, and the interaction of drawing series with condition, $F(8,70)=3.01, p<$ $.01, \eta^{2}=.10$, remained significant (see Figure 7A).

17-year-olds. There were no participants of this age group who used crayons. The main effects of the drawing series, $F(3.46,62)=23.22, p<.001, \eta^{2}=.28$, and condition, $F(2$, $62)=4.90, p<.05, \eta^{2}=.14$, were significant and interacted with each other, $F(8,62)=3.09, p<.01, \eta^{2}=.10$ (see Figure $6 \mathrm{D})$. Post hoc tests showed that size variability in the practice condition was significantly larger than in the random condition (Spatial Systems 3 and 5) and in the logical sequence condition (Spatial System 3).

When the same model was run without the bird's-eye view drawers, only the effect of the experimental condition remained significant, $F(2,47)=3.52, p<.05, \eta^{2}=.14($ see Figure $7 \mathrm{~B})$, whereas the significance of all other effects disappeared $(p s>$ 
A

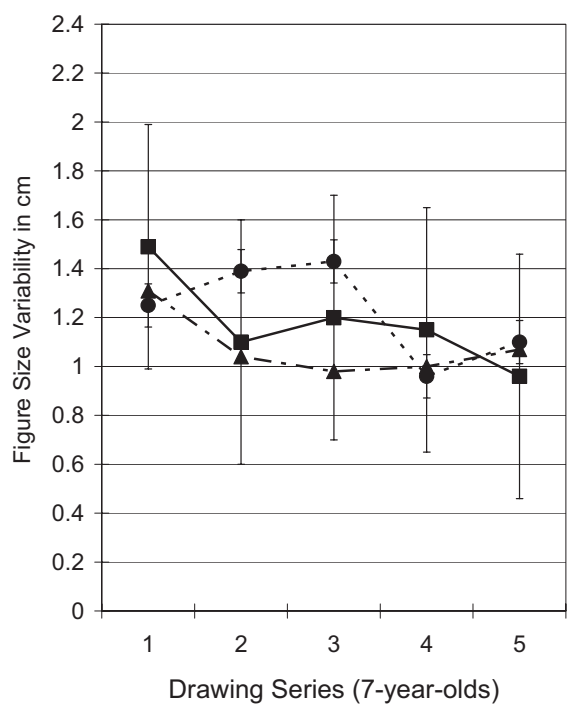

C

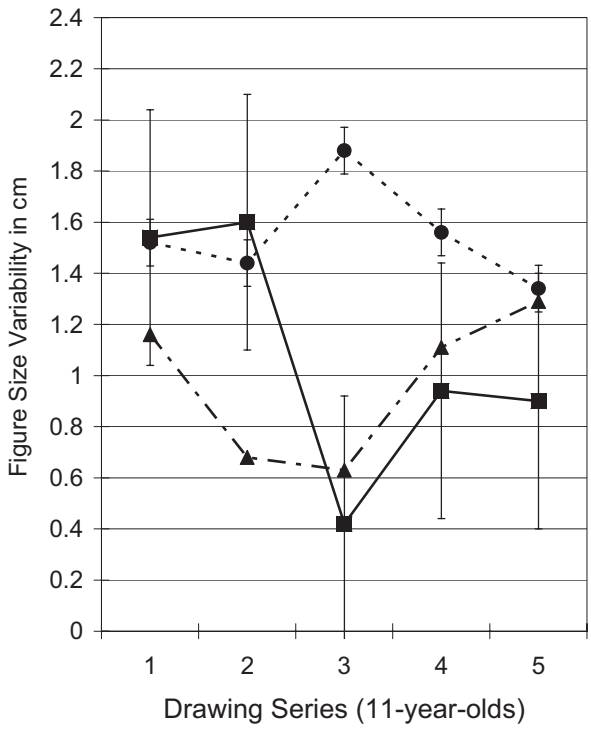

logical
- $\mathbf{-}$ - random

practice

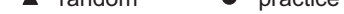
(n)

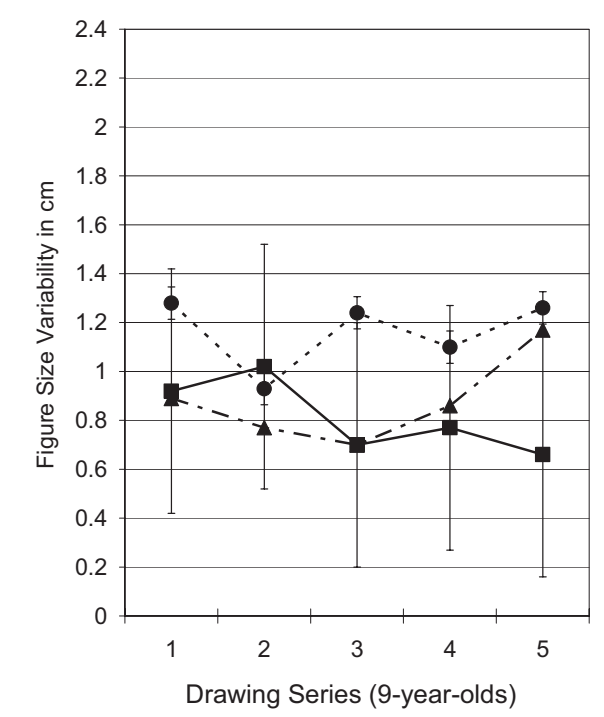

logical

\section{D}

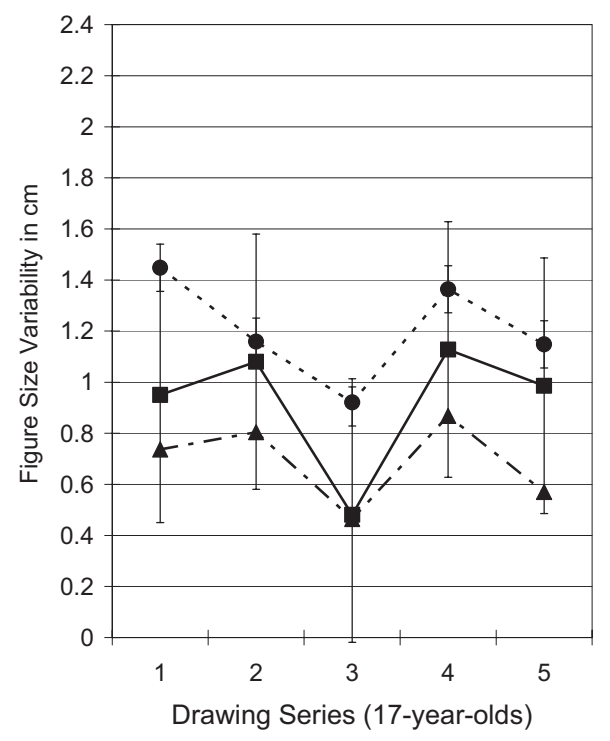

logical

ractice

Figure 6. Figure size variability: Plotted means of the three-way interaction between age groups, drawing series, and condition. (A) 7-year-olds, (B) 9-year-olds, (C) 11-year-olds, and (D) 17-year-olds. From 9 years of age, crossed axes systems elicited significantly less size variation, whereas Space Systems 1 and 2, with less spatial constraints, and the practice condition generated larger figure size variability.

.13). This showed that in late adolescents, the random sequence of space systems not only had the greatest impact on reducing figure size but also reduced figure size variability, whereas the practice condition with empty sheets allowed for larger figures and increased figure size variability.

\section{Discussion}

The present study showed that, as predicted, figure size and figure size variability would be smaller in orthogonal and diagonal axes systems. The results replicated those of studies using free 
A

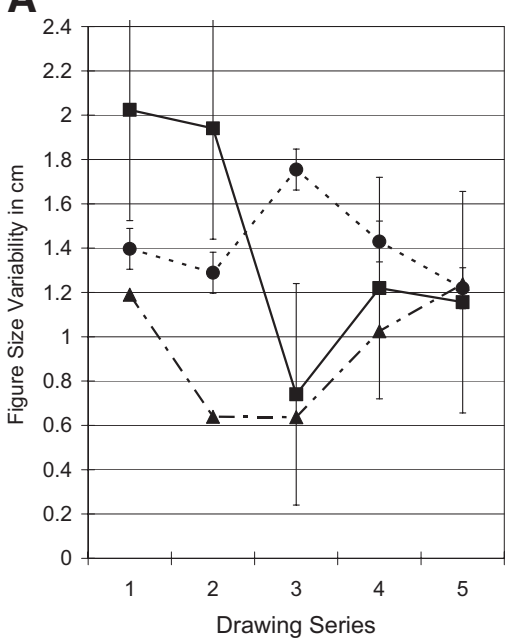

(11-year-olds, no bird's-eye view drawers)
B

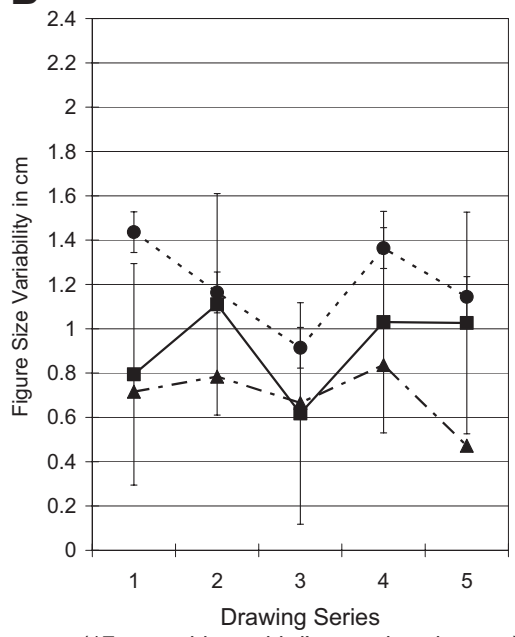

(17-year-olds, no bird's-eye view drawers)

- logical -\$-random - - practice

Figure 7. Figure size variability without the bird's-eye view drawers. (A) 11-year-olds and (B) 17-year-olds.

drawing tasks as well as ready-made axes systems (Lange-Küttner, 1997, 2004)

The gradual increase in complexity of spatial axes systems, which followed the developmental acquisition sequence, was essential for figure size reduction especially in 7-year-olds. The comparison with the practice and random control conditions revealed, in addition, that the ready-made three-dimensional space systems were not yet powerful enough frameworks to modify the embedded objects beyond the average figure size of the other two experimental conditions nor did the randomized sequence produce similar results as the logical sequence of spatial systems. For this reason, one might want to explain that size reduction in 7-year-olds in the logical sequence resulted from a perceptual "pull" of the dimensional space system transitions in the drawing series to which the children responded (see also Plumert, Hund, \& Recker, 2007).

Nine-year-old children reduced figure size in the logical and the random condition at the same level, and, importantly, the practice condition for the first time yielded significantly and considerably larger figures on average. The empty, unstructured white pages of paper afforded the drawer with many more degrees of freedom to expand figure size, which the 7-year-olds did not use. This was quite astonishing because researchers using a planning approach to explain the absence of realistic proportion in younger children's drawings demonstrated that such children tended to start too large (Freeman, 1980; Thomas, 1995). However, this concerned younger children and proportion within one figure (head-trunk ratio) or between two figures (dog-owner size ratio). In the current study, figure size was measured in response to an overall space system. It could be the case that 7-year-olds were not actually using this framework for planning but were drawing figures at a habitual size, that is, that they would draw a figure in a size that was independent of how much available area there was in each space system. In other words, the figures were not yet projections in space.
The emerging sensitivity to bounded area in 9-year-olds was also apparent in another recent study, which used a nearly reversed drawing task, the Common Region Test (CRT). In the CRT, three lines of dots were predrawn (see also Palmer, 1992; Wertheimer, 1923), and children were asked to draw spatial boundaries (circles) around those shapes that belonged together (Lange-Küttner, 2006). Young children would assign one place to each shape, but at 9 years, most children would draw spatial boundaries around pairs that were either similar in appearance or close in proximity, that is, they would no longer assign individual places for "spatially isolated" objects (see also Tada \& Stiles, 1996), but also a "common area" (Lange-Küttner, 2006; Palmer, 1992). Hence, children at this age might have used the entire area of the drawing sheet for planning their figure drawings.

The question of when and how children conceptualize area has been a long-standing issue in developmental psychology (e.g. Gigerenzer, 1984; Gigerenzer \& Richter, 1990; Wilkening, 1979), but this question previously was concerned with the judgment of the size of an area within the boundary of one shape or the relative area of two bounded shapes, not an area segmented in space. The beginning of explicit axes systems does not start off in a unit-based way with a graphic shape for a tangible, concrete object entity, such as a cube, but rather with a frame-based strategy involving initially horizontal axes. Explicit spatial axes systems conceptualize empty space, as in astronomy, where the extension of the universe is conceptualized with reference frames (Lange-Küttner, 2008c). In the absence of spatial boundaries, children of this age find it very hard to associate objects with regions, whereas this is much less of a problem for adults (Plumert et al., 2007), for whom reference frames are the "most powerful and stable "attractor" (Vinter \& Marot, 2007, p. 108). One might argue that a horizontal axes system still denotes the ground we are standing on, and an orthogonal axes system was still a playing field, all relatively real-life areas that are concrete rather than abstract reference frames. However, these kinds of areas also need explicit bound- 
aries in space, like differently paved and colored areas for pedestrians versus cars, internal versus external field boundaries on football lawns, and so forth. The current ready-made axes systems method did not fully permit a determination of whether children would use the spatial fields in the orthogonal and diagonal systems on a concrete or an abstract processing level. On the one hand, no area was marked with a concrete surface property, such as color or pattern, and in the open diagonal system, the spatial axes did point beyond the drawing sheet like vectors, so that one could see the axes systems as relatively high-level and abstract. On the other hand, in the instructions, children were explicitly told that the axes delineated the spatial boundaries of a playing field, and they were required to draw the players. Hence, instructions would have guided children's perception toward seeing the axes systems more as spatial field boundaries, as in direct perception of the real-life visual flow. To systematically disentangle and compare whether a reference frame is an abstract grid that acts like a layer dividing the percept into quadrants or an abstract vector system denoting direction and extension-or whether a reference frame denotes the rather concrete spatial boundaries of a spatial field of any size (i.e., also one that extends beyond the boundaries of the drawing sheet), another experiment would be required.

One could say, however, that without awareness of spatial constraints and possibilities (see also Piaget, 1981, 1983; Smith, 1993), there may have been no exploitation of the degrees of freedom for a larger size depiction on the empty sheets in the practice condition. Hence, even if the level of processing of the axes systems may not be entirely clear, the control condition on the empty sheets indicates that some awareness, rather than mere direct perception, of spatial axes had emerged in the 9-year-olds. Thus far, it may be concluded that 7-year-olds appeared to be sensitive to the perceptual pull of the gradual and logically increasing dimensional space system transitions, whereas 9-yearolds became sensitive to the degrees of freedom for figure size depiction within an area.

Whereas 11-year-olds also showed increased size in the control practice condition, space systems had now become more powerful in their impact on the embedded figures. In the logical sequence, children were drawing figures on the empty page and in the horizontal axes system as large as in the practice condition; while in the crossed axes systems, children drew figures as small as in the random sequence condition. Children were now also using a larger spatial scale than were the 9-year-olds, and this was particularly the case in the bird's-eye view drawers, who would draw very small in the closed space of the orthogonal spatial field and very large in empty space. Thus, that bird's-eye view drawers were drawing smaller in the orthogonal system because of the change in view should not be seen as deviant, first, because also some of the remaining children were drawing small in this condition; second, because statistical effects showing size reduction in the crossed axes systems stayed significant without this subgroup; and last but not least, because drawing a top view in the orthogonal spatial field is a sign of maturity, as these children were able to change the perspective on figures.

Thus, one could summarize that 11-year-olds could make use of a larger spatial scale. In addition, they were definitely no longer being pulled into the dimensional space system transitions of the drawing series as demonstrated by a now nearly identical figure size in the crossed axes systems of both the logical and the random sequence. However, a difference between these two conditions also emerged, as average figure size in the space systems with more degrees of freedom (empty and horizontal axes systems) was much smaller in the random than in the logical sequence. Because the empty sheet or a horizontal axes system with few spatial constraints could be encountered anytime in the random sequence, it appears that prior experience with a more constrained explicit space system may have primed small figure size in the less constrained space systems. Vinter and Marot (2007) showed that priming with a spatial reference frame improved children's drawing of a staircase. It would be interesting to see what would happen if the logical sequence of space systems had been given in the reverse order. In fact, in an earlier study (Lange-Küttner, 2004), when drawing five friends, 7-year-olds who constructed explicit spatial axes on the first page of the drawing series produced the smallest figures and did not reduce size any further thereafter. Thus, in conclusion, spatial constraints leading to size reduction had become more powerful than spatial possibilities leading to size expansion at age eleven.

Basically, the same results were obtained for the 17-year-olds as for the 11-year-olds. In addition, there was a trend in which late adolescents showed a subtle increase in figure size with more available area in the perspective systems; however, late adolescents who drew in bird's-eye view no longer showed the huge figure size expansion on the empty sheet in the logical sequence. This pointed to a further development toward more powerful spatial constraints, which may indicate a beginning development toward adults' proclivity to judge visual percepts exclusively in terms of area and proportion (McManus, 1980).

That spatial constraints on figure size became more powerful may have occurred for a good reason. What happens in drawing development is that the child has good three-dimensional vision of object surfaces from early on (Spelke, 1990), yet initially creates two-dimensional drawings (Kosslyn et al., 1980). To produce a two-dimensional to three-dimensional transformation in pictorial space, the child must modify the initial human figure drawing consisting of aggregates of picture primitives of circles with extensions into an object with a natural contour (Lange-Küttner, 2008c; Lange-Küttner et al., 2002; Willats, 1985, 1997, 2005). Einstein (1920/1993) thought of objects in space systems as nonrigid reference bodies, which he termed "reference-molluscs" (p. 99), as space could only be conceptualized as a truly homogeneous expanse if every point on an object were treated as a space point. Modern computer graphics conceptualize objects as small local axes systems, which are embedded in a larger, global world axes system (Hill \& Kelley, 2007). To model object changes in space, the object's local coordinates are transformed according to the object's world coordinates, with processes such as spatial translation, rotation, and scaling. The multitude of spatial processes involved in an object modification could also be demonstrated for children's drawing of rotated objects (Lange-Küttner \& Green, 2007).

Hence, if one conceptualized the height of figures as the $y$-axis of the object's local coordinate within the pictorial world coordinates, then an oversized figure such as a portrait would tend to replace the pictorial world coordinate system as an overarching system. Thus, in the current task, constraining object size would be more important than expanding object size, if a power balance and 
a sense of proportion between objects and space are to be maintained in a local-global nested axes system.

In summary, 7-year-olds were sensitive toward the perceptual pull of the gradual dimensional increase in the transitions between space systems but showed some habitual, average figure size in drawing that did not differ between drawing conditions. After the age of 9 years, an emergence of sensitivity toward spatial constraints and possibilities (degrees of freedom) functioned as an important mechanism toward a more powerful pictorial space concept. Spatial constraints became increasingly more powerful so as to create and maintain proportion between objects and pictorial space. Furthermore, space systems did not have to gradually unfold in dimensionality but could remain in a randomized sequence, that is, older children did not need the pull of this gradual process but could flexibly respond to the spatial constraints and possibilities of each space system in turn. The emergence of this ability to control randomly appearing scenes may be useful for future research into the question of how much children and young adults are drawn into virtual reality simulations.

From a wider theoretical perspective, therefore, one may not necessarily agree with the view that children would change from an 'impoverished' concept to a "richer" concept (PiatelliPalmarini, 1980, p. 149), as at 7 years of age, children simultaneously understood all space systems and, hence, may have a rich space concept. This is probably the case because children of this age can use the experience of their own changing viewpoints in the visual flow when manipulating drawing cues, such as height-inpictures (Bremner \& Andreasen, 1997). One could explain the developmental change from the importance of visual flow in young children to area constraints in older children with the core tenets of two separate learning systems (Keele, Ivry, Mayr, Hazeltine, \& Heuer, 2003). Keele et al. assumed a multidimensional learning system, which builds associations between events from different modalities, whereas a unidimensional learning system would be restricted to information processing along a single dimension. With respect to drawing figures in pictorial space systems, one could thus assume that 7-year-old children use a multidimensional learning system, which draws on visual flow information from the environment for drawing, whereas from the age of 9 years, children may tend to use a unidimensional learning system insofar as drawing domain-specific properties of area and spatial boundaries in pictorial space become considered. Also in some other drawing research, individual differences in 7-year-old children from different environments (agrarian communities = extended landscape $=$ small figures; service and trade communities $=$ built environment $=$ large figures) were replaced by domain-specific pictorial factors, such as the number of figures drawn and the level of axes system used by 9- and 11-year-olds (Lange-Küttner, 1997, 2008c).

However, the study did find evidence for a change from a weaker to a more powerful cognitive system, a question that was much debated in the 1980s (Piatelli-Palmarini, 1980, pp. 142). Fodor claimed that a conceptual transition could be explained only by maturation, or adherence to expectations of teachers, whereas Piaget held that the weaker system in itself would already contain the possibilities for further conceptual development. In the current study, this was borne out by the changes in children's visual cognition after the age of 7 years. One could say that the counterintuitive combination of a rich, but weak spatial concept at 7 years of age becomes more powerful and flexible through the new emerging sensitivity toward spatial constraints and spatial possibilities of bounded area, that is, a process that one could call two-dimensional viewing. One could even presume that whereas 7-year-olds were actually seeing perspective in the more complex axes systems, older children, when assessing constraints and possibilities, may have performed a perceptual transformation from a three-dimensional perspective to a two-dimensional area. Further research is needed to test whether a three-dimensional to twodimensional transformation in looking may be part of the ability to construct a two-dimensional to three-dimensional transformation in drawing.

That this changing sensitivity could be revealed despite the fact that the children did not have to construct diagonal perspective themselves may speak of a good deal of implicit learning in development. However, the observation that children often do not achieve their full potential when having to construct diagonal perspective themselves, which requires effort, that is, planning, memory, and strategy, points to an overestimation of selforganization of cognition in children, who might need some scaffolding while in a zone of proximal development (see Lloyd \& Fernyhough, 1999; Stetsenko, 1995).

The present study focused on the depiction of size within space systems, which, of course, provides only a limited view on the development of the pictorial space concept. Other variables such as figure orientation (Lange-Küttner \& Green, 2007) could be measured. The current analysis of size and size variability in this highly controlled experimental design showed how figures could become stretched or shrunk along with the pictorial space systems. In this way, size appeared to be an excellent marker variable, which has been highly informative about an emerging sensitivity toward spatial degrees of freedom as a generator for the development of a more powerful pictorial space concept.

\section{References}

Barlow, C. M., Jolley, R. P., White, D. G., \& Galbraith, D. (2003). Rigidity in children's drawings and its relation with representational change. Journal of Experimental Child Psychology, 86, 124-152.

Bremner, J. G., \& Andreasen, G. (1997). A change in viewpoint promotes the use of height-in-picture as a depth cue in 5- to 7-year-olds' drawings of a simple depth relationship. Journal of Experimental Child Psychology, 66, 180-192.

Bremner, J. G., Morse, R., Hughes, S., \& Andreasen, G. (2000). Relations between drawing cubes and copying line diagrams of cubes in 7 - to 10-year-old children. Child Development, 71, 621-634.

Caron-Pargue, J. (1992). A functional analysis of decomposition and integration in children's cylinder drawing. British Journal of Developmental Psychology, 10, 51-69.

Case, R., Stephenson, K. M., Bleiker, C., \& Okamoto, Y. (1996). Central spatial structures and their development. In R. Case \& Y. Okamoto (Eds.), The role of central conceptual structures in the development of children's thought. Monographs of the Society for Research in Child Development, 61(1-2, Serial No. 246).

Cox, M. V. (1986). Cubes are difficult things to draw. British Journal of Developmental Psychology, 4, 341-345.

Davis, A. (1983). Contextual sensitivity in young children's drawings. Journal of Experimental Child Psychology, 35, 478-486.

Davis, A. (1985). Conflict between canonicality and array-specificity in young children's drawings. British Journal of Developmental Psychology, 3, 363-372. 
Dennis, S. (1992). Stage and structure in the development of children's spatial representations. In R. Case (Ed.), The mind's staircase (pp. 229-245). Hillsdale, NJ: Erlbaum.

Deregowski, J. B. (1978). On reexamining Fortes' data: Some implications of drawings made by children who have never drawn before. Perception, 7, 479-484.

Deregowski, J. B. (1980). Experienced and inexperienced model makers: Model-making and -drawing in Rhodesia. Journal of Cross-Cultural Psychology, 11, 189-202.

Deregowski, J. B. (2008). On contours seen and contours drawn. In C. Lange-Küttner \& A. Vinter (Eds.), Drawing and the non-verbal mind: A life-span perspective (pp. 239-257). Cambridge, United Kingdom: Cambridge University Press.

Deregowski, J. B., \& Dziurawiec, S. (1996). The puissance of typical contours and children's drawings. Australian Journal of Psychology, 48, 98-103.

Deregowski, J. B., \& Parker, D. M. (1996). The depiction of distance: A Bartelian analysis. Perception, 25, 177-185.

Dubery, F., \& Willats, J. (1972). Perspective and other drawing systems. New York: Van Nostrand.

Ebersbach, M., \& Resing, W. C. M. (2007). Shedding new light on an old problem: The estimation of shadow sizes in children and adults. Journal of Experimental Child Psychology, 97, 265-285.

Einstein, A. (1993). Relativity: The special and the general theory (R. W. Lawson, Trans.). London, United Kingdom: Routledge. (Original work published 1920

Fisher, R. A., \& Yates, F. (1963). Statistical tables for biological, agricultural and medical research. Edinburgh, United Kingdom: Oliver \& Boyd.

Freeman, N. H. (1980). Strategies of representation in young children. London, United Kingdom: Academic Press.

Gigerenzer, G. (1984). Lässt sich die Flächenwahrnehmung als 'kognitive Algebra' beschreiben? [Can we model area perception by 'cognitive algebra'?]. Psychologische Beiträge, 26, 113-119.

Gigerenzer, G., \& Richter, H. R. (1990). Context effects and their interaction in development: Area judgments. Cognitive Development, 5, 235-264.

Hagen, M. A. (1985). There is no development in art. In N. Freeman \& M. V. Cox (Eds.), Visual order (pp. 78-100). Cambridge, United Kingdom: Cambridge University Press.

Hargreaves, D. J., Jones, P. M., \& Martin, D. (1981). The air gap phenomenon in children's landscape drawings. Journal of Experimental Child Psychology, 32, 11-20.

Hill, F. S., Jr., \& Kelley, S. M. (2007). Computer graphics using open GI (3rd ed.). Upper Saddle River, NJ: Prentice Hall.

Karmiloff-Smith, A. (1990). Constraints on representational change: Evidence from children's drawings. Cognition, 34, 57-83.

Karmiloff-Smith, A. (1995). Beyond modularity: A developmental perspective on cognitive science. Cambridge, MA: MIT Press.

Keele, S. W., Ivry, R. B., Mayr, U., Hazeltine, E., \& Heuer, H. (2003). The cognitive and neural architecture of sequence representation. Psychological Review, 110, 316-339.

Kosslyn, S. M. (1994). Image and brain. Cambridge, MA: MIT Press.

Kosslyn, S. M., Heldmeyer, K. H., \& Locklear, E. P. (1980). Children's drawings as data about their internal representations. Journal of Experimental Child Psychology, 23, 191-211.

Lange-Küttner, C. (1989). Raumbegriff und Objektbeziehungen beim Kind [Space concept and object relations in the child]. Frankfurt/Main, Germany: Lang.

Lange-Küttner, C. (1994). Gestalt und Konstruktion [Gestalt and construction]. Bern, Switzerland: Huber.

Lange-Küttner, C. (1997). Development of size modification of human figure drawings in spatial axes systems of varying complexity. Journal of Experimental Child Psychology, 66, 264-278.
Lange-Küttner, C. (1998). Pressure, velocity and time in speeded drawing of basic graphic pattern by young children. Perceptual and Motor Skills, $86,1299-1310$.

Lange-Küttner, C. (2004). More evidence on size modification in spatial axes systems of varying complexity. Journal of Experimental Child Psychology, 88, 171-192.

Lange-Küttner, C. (2006). Drawing boundaries: From individual to common region-The development of spatial region attribution in children. British Journal of Developmental Psychology, 24, 419-427.

Lange-Küttner, C. (2008a, July). Different repetition/training effects in spatial memory and drawing. In A. Vinter \& C. Lange-Küttner (Chairs), Perception, action and graphic representation. Symposium conducted at the 29th International Congress of Psychology, Berlin, Germany.

Lange-Küttner, C. (2008b). Figures in and out of context: Absent, simple, complex and halved spatial fields. In C. Lange-Küttner \& A. Vinter (Eds.), Drawing and the non-verbal mind: A life-span perspective (pp. 199-220). Cambridge, United Kingdom: Cambridge University Press.

Lange-Küttner, C. (2008c). Size and contour as crucial parameters in children drawing images. In C. Milbrath \& H.-M. Trautner (Eds.), Children's understanding and production of pictures, drawing, and art-Theoretical and empirical approaches (pp. 89-106). Göttingen, Germany: Hogrefe.

Lange-Küttner, C., \& Green, H. (2007). What is the age of mental rotation? Proceedings of the 6th IEEE International Conference on Development and Learning, 6, 259-263.

Lange-Küttner, C., Kerzmann, A., \& Heckhausen, J. (2002). The emergence of visually realistic contour in the drawing of the human figure. British Journal of Developmental Psychology, 20, 439-463.

Lange-Küttner, C., \& Reith, E. (1995). The transformation of figurative thought: Implications of Piaget and Inhelder's developmental theory for children's drawings. In C. Lange-Küttner \& G. V. Thomas (Eds.), Drawing and looking (pp. 75-92). Hemel Hampstead, United Kingdom: Harvester Wheatsheaf/Pearson.

Lange-Küttner, C., \& Vinter, A. (2008). Contemporary enquiries into a long-standing domain: Drawing research. In C. Lange-Küttner \& A. Vinter (Eds.), Drawing and the non-verbal mind: A life-span perspective (pp. 1-20). Cambridge, United Kingdom: Cambridge University Press.

Light, P. H., \& Humphreys, J. (1981). Internal spatial relationships in young children's drawings. Journal of Experimental Child Psychology, $31,521-530$

Light, P. H., \& MacIntosh, E. (1980). Depth relationships in young children's drawings. Journal of Experimental Child Psychology, 30, 79-87.

Light, P. H., \& Nix, C. (1983). 'Own view' versus 'good view' in a perspective-taking task. Child Development, 54, 79-87.

Lloyd, P., \& Fernyhough, C. (1999). Lev Vygotsky: Critical assessments: Vol. 3.: The zone of proximal development. London, United Kingdom: Routledge.

Luquet, G.-H. (1927). Le dessin enfantin [Children's drawings]. Paris: Alcan.

Luquet, G.-H. (2001). Children's drawings (A. Costall, Trans.). London: Free Association Books. (Original work published 1927)

McManus, I. C. (1980). The aesthetics of simple figures. British Journal of Psychology, 71, 505-524.

Mitchell, P., \& Taylor, L. M. (1999). Shape constancy and theory of mind: Is there a link? Cognition, 70, 167-190.

Mitchelmore, M. C. (1978). The developmental stages in children's representation of regular solid figures. Journal of Genetic Psychology, 133, 229-239.

Moore, V. (1986). The relationship between children's drawings and preferences for alternative depictions of a familiar object. Journal of Experimental Child Psychology, 42, 187-198.

Morra, S. (2008). Children's spatial structures in children's drawings: How do they develop? In C. Lange-Küttner \& A. Vinter (Eds.), Drawing and 
the non-verbal mind: A life-span perspective (pp. 164-198). Cambridge, United Kingdom: Cambridge University Press.

Nicholls, A. (1995). Influence of visual projection on young children's depictions of object proportions. Journal of Experimental Child Psychology, 60, 304-326.

Olson, D. R. (1996). Cognitive development: The child's acquisition of diagonality. Mahwah, NJ: Erlbaum. (Original work published 1970)

Palmer, S. E. (1992). Common region: A new principle of perceptual grouping. Cognitive Psychology, 24, 436-447.

Perner, J., Kohlmann, R., \& Wimmer, H. (1984). Young children's recognition and use of the vertical and horizontal in drawings. Child Development, 55, 1637-1645.

Piaget, J. (1981). Le possible et le nécessaire I. L'evolution des possibles chez l'enfant [The possible and the necessary in the child. I. The evolution of the possible in the child]. Paris: Presses Universitaires de France.

Piaget, J. (1983). Le possible et le nécessaire II. L'evolution du nécessaire chez l'enfant [The possible and the necessary in the child. II. The evolution of the necessary in the child]. Paris: Presses Universitaires de France.

Piaget, J., \& Inhelder, B. (1956). The child's conception of space. London: Routledge \& Kegan Paul.

Piaget, J., Inhelder, B., \& Szeminska, A. (1960). The child's conception of geometry. London: Routledge \& Kegan Paul.

Piatelli-Palmarini, M. (1980). Language and learning. Cambridge, MA: Harvard University Press.

Picard, D., \& Vinter, A. (1999). Representational flexibility in children's drawings: Effects of age and verbal instructions. British Journal of Developmental Psychology, 17, 605-622.

Picard, D., \& Vinter, A. (2007). Relationships between procedural rigidity and interrepresentational change in children's drawing behavior. Child Development, 78, 522-541.

Plumert, J. M., Hund, A. M., \& Recker, K. M. (2007). Organismenvironment interaction in spatial development. Explaining categorical bias in memory for location. In J. Plumert \& J. P. Spencer (Eds.), The emerging spatial mind (pp. 25-52). Oxford, United Kingdom: Oxford University Press.

Reith, E. (1988). The development of use of contour lines in children's drawings of figurative and non-figurative three-dimensional models. Archives de Psychologie, 56, 83-103.

Silk, A. M. J., \& Thomas, G. V. (1986). Development and differentiation in children's figure drawings. British Journal of Psychology, 77, 399-410.

Silk, A. M. J., \& Thomas, G. V. (1988). The development of size scaling in children's figure drawings. British Journal of Developmental Psychology, 6, 285-299.

Smith, L. (1993). Necessary knowledge. Hove, United Kingdom: Erlbaum. Spelke, E. S. (1990). Principles of object perception. Cognitive Science, 14, $29-56$.

Stetsenko, A. (1995). The psychological function of children's drawings: A
Vygotskian perspective. In C. Lange-Küttner \& G. V. Thomas (Eds.), Drawing and looking (pp. 147-158). Harlow, United Kingdom: Pearson.

Tada, W. L., \& Stiles, J. (1996). Developmental change in children's analysis of spatial patterns. Developmental Psychology, 32, 951-970.

Thomas, G. V. (1995). The role of drawing strategies and skills. In C. Lange-Küttner \& G. V. Thomas (Eds.), Drawing and looking (pp. 107-122). Harlow: Pearson.

Van Sommers, P. (1984). Drawing and cognition: Descriptive and experimental studies of graphic production processes. Cambridge, United Kingdom: Cambridge University Press.

Vinter, A., \& Marot, V. (2007). The development of context sensitivity in children's graphic copying strategies. Developmental Psychology, 43, 94-110.

Vinter, A., \& Perruchet, P. (2000). Implicit learning in children is not related to age: Evidence from drawing behavior. Child Development, 71, $1223-1240$

Vinter, A., \& Perruchet, P. (2002). Implicit motor learning through observational training in adults and children. Memory \& Cognition, 30, $256-261$.

Vinter, A., Picard, D., \& Fernandes, V. (2008). Graphic syntax and representational development. In C. Lange-Küttner \& A. Vinter (Eds.), Drawing and the non-verbal mind: A life-span perspective (pp. 145163). Cambridge, United Kingdom: Cambridge University Press.

Wertheimer, M. (1923). Untersuchungen zur Lehre von der Gestalt [Studies on the teachings of the Gestalt]. Psychologische Forschung, 4, 301-350.

Wilkening, F. (1979). Combining of stimulus dimensions in children's and adults' judgments of area: An information integration analysis. Developmental Psychology, 15, 25-33.

Willats, J. (1985). Drawing systems revisited: The role of denotation systems in children's figure drawings. In N. H. Freeman \& M. V. Cox (Eds.), Visual order (pp. 78-100). Cambridge, MA: Cambridge University Press.

Willats, J. (1997). Art and representation: New principles in the analysis of pictures. Princeton, NJ: Princeton University Press.

Willats, J. (2005). Making sense of children's drawings. Mahwah, NJ: Erlbaum.

Wilson, M., \& Wilson, B. (1982). The case of the disappearing of the two-eyed profile or how little children influence the drawings of little children. Review of Research in Visual Arts Education, 15, 19-32.

Zhang, Z., Thomas, G. V., \& Robinson, E. J. (1997). Constraints on representational change: Drawing a man with two heads. British Journal of Developmental Psychology, 15, 275-290.

Received October 26, 2006

Revision received November 6, 2008 Accepted February 23, 2009 